\title{
Predicting the risk of parafoveal scotoma in myopic normal tension glaucoma: role of optic disc tilt and rotation
}

\author{
MS Sung ${ }^{1}, \mathrm{H} \mathrm{Heo}{ }^{1}$, YS Ji ${ }^{1}$ and SW Park ${ }^{1,2}$
}

\begin{abstract}
Purpose The purpose of the study was to evaluate the factors associated with development of parafoveal scotoma in early myopic normal tension glaucoma (NTG). Patients and methods Ninety-nine myopic NTG patients with mean deviation (MD) $>-6.0$ decibels $(\mathrm{dB})$ were enrolled.

Parafoveal scotoma was defined as a visual field (VF) defect within $10^{\circ}$ of fixation with at least one point at $P<1 \%$ lying at the four innermost central points. Systemic factors, optic disc characteristics including tilt ratio, rotation degree, $\beta$-zone parapapillary atrophy, disc hemorrhage, and peripapillary retinal nerve fiber layer and macular ganglion cellinner plexiform layer (mGCIPL) thickness parameters using optical coherence tomography were evaluated. Logistic regression analysis was performed to identify factors associated with the development of parafoveal scotoma.

Results The mean spherical equivalent refractive error and MD were $-6.07 \pm 2.83$ diopters and $-3.29 \pm 1.70 \mathrm{~dB}$, respectively. Among 99 eyes, 42 (42.42\%) showed parafoveal scotoma. Eyes with parafoveal scotoma had greater disc tilt, lesser disc rotation, lower $\mathrm{MD}$, thinner minimum mGCIPL, and a higher proportion of VF defect in the superior hemifield than eyes without parafoveal scotoma. Multivariate logistic regression showed that all these parameters were significantly associated with development of parafoveal scotoma $(P=0.047$, $P=0.011, P=0.032, P=0.010$, and $P=0.001$, respectively).

Conclusion In addition to the previously reported risk factors, optic disc characteristics, such as tilt ratio and optic disc rotation, were also significantly associated with development of parafoveal scotoma in patients with myopic NTG.
\end{abstract}

Eye (2017) 31, 1051-1059; doi:10.1038/eye.2017.33; published online 10 March 2017

\section{Introduction}

Parafoveal scotoma is an important concern in glaucoma patients because of its proximity to fixation. ${ }^{1-5}$ Several factors have been reported to be associated with parafoveal scotoma in early glaucoma, including systemic hypotension, migraine, Raynaud's phenomenon, sleep apnea, autonomic dysfunction, ocular pulse amplitude, and disc hemorrhage. 3,6,7 These factors seem to be related to systemic hemodynamic or vascular dysregulation, and ultimately to unstable ocular perfusion, which acts as one of the important pathomechanisms of normal tension glaucoma (NTG). It has been postulated that mechanisms independent of intraocular pressure (IOP) may play a relatively larger role in the onset of glaucomatous optic nerve damage in individuals with NTG, and eyes with NTG tend to show more localized and parafoveal visual field (VF) defects than eyes with high tension glaucoma. ${ }^{8-11}$

In myopic eyes, morphological changes in the optic disc induced by axial elongation, such as $\beta$-zone parapapillary atrophy (PPA), tilt, and rotation, have an important role in the development of glaucoma. ${ }^{12-15}$ However, little is known about their relationship to the pattern of VF defects. Although, several reports identified anatomic factors that can influence the development of parafoveal scotoma in early glaucoma, ${ }^{16,17}$ the subjects included in these studies were NTG patients and not confined to the myopic subpopulation. From a clinical perspective, patients with myopic NTG have been reported to be more susceptible to papillomacular bundle damage, which is associated with parafoveal scotoma, and the pathomechanism of optic nerve damage in eyes with myopic NTG might be different from that
${ }^{1}$ Department of Ophthalmology and Research Institute of Medical Sciences, Chonnam National University Medical School and Hospital, Gwangju, South Korea

${ }^{2}$ Center for Creative Biomedical Scientists, Chonnam National University, Gwangju, South Korea

Correspondence: SW Park, Department of Ophthalmology and Research Institute of Medical Sciences, Chonnam National University Medical School and Hospital, 42 Jebong-ro, Gwangju 61469, South Korea Tel: +82 62 2206742; Fax: +82 622271642 E-mail: exo70@naver.com

Received: 8 October 2016 Accepted in revised form: 17 January 2017 Published online: 10 March 2017 
in eyes with non-myopic NTG. ${ }^{18}$ Considering the previous report by Park et al ${ }^{15}$ that compared the clinical characteristics of myopic NTG with those of non-myopic NTG patients and demonstrated the significant differences in optic disc tilt, rotation, and PPA between the two groups, we hypothesized that myopic optic disc morphology may give a clue to understand the high prevalence of parafoveal scotoma in myopic NTG. Hence, the present study was designed to evaluate the factors associated with the development of parafoveal scotoma in eyes with myopic NTG, with a focus on optic disc characteristics such as optic disc tilt and rotation.

\section{Materials and methods}

\section{Subjects}

A retrospective analysis was performed on 99 eyes of 99 consecutive glaucoma subjects who were diagnosed with early myopic NTG in the glaucoma clinic at Chonnam National University Hospital from January 2013 to December 2015. At baseline examination, the diagnosis of NTG was based on the presence of glaucomatous optic neuropathy, corresponding abnormal 30-2 Swedish Interactive Thresholding Algorithm (SITA) standard automated perimetry examinations (Humphrey Field Analyzer; Carl Zeiss Meditec Inc., Dublin, CA, USA), open anterior chamber angles by gonioscopy, no identifiable secondary cause of glaucoma, and all known untreated IOPs $\leq 21 \mathrm{~mm} \mathrm{Hg}$ by Goldmann applanation tonometry. Glaucomatous optic nerve damage was defined as a vertical cup-to-disc ratio $\geq 0.7$, asymmetry in the vertical cup-to-disc ratio between both eyes of more than 0.2 , the presence of focal neural rim notching, or generalized loss of the neural rim on disc photography and red-free retinal nerve fiber layer (RNFL) photography (Canon, Tokyo, Japan).

In order to be included in the study, all subjects with myopic NTG had to meet the following criteria: 18 years of age or older, best-corrected visual acuity better than $20 / 40$, spherical equivalent (SE) refractive error $\leq-3.0$ diopters (D), astigmatism within $\pm 2.0 \mathrm{D}$, and a mean deviation (MD) value better than -6.0 decibels $(\mathrm{dB})$. A SE refractive error $<-6.0 \mathrm{D}$ was defined as high myopia. In addition, all subjects had to be followed for at least 2 years in our clinic or local eye clinic before the enrollment. We only included the subjects who had showed the progressive glaucomatous VF defect and optic nerve damage during the follow-up period. Patients with a history of intraocular or refractive surgery, pathologic myopia (patch chorioretinal atrophy, lacquer crack lesions, intrachoroidal cavitations, an abrupt change in the scleral curvature temporal to the optic disc, or choroidal neovascularization), other evidence of retinal pathology, opaque media such as cataract, previous or current use of systemic or topical steroids, or any history of neurological disease leading to a VF abnormality were excluded. Patients with optic disc suspicious for congenital disc anomaly were excluded. Patients with a horizontally oval disc and situs inversus of the retinal vessels, suggestive of tilted disc syndrome, were also excluded from the analysis. Eligibility was determined by two glaucoma specialists (SWP and MSS), who evaluated the appearance of the optic disc on stereoscopic disc photographs, RNFL defects on red-free fundus photographs, and the results of VF examinations. Evaluators were masked to all other patient and ocular data, and an eye was excluded from the study analyses if a consensus could not be reached. When both eyes satisfied the inclusion criteria, only one randomly selected eye from each participant was included in the study. The study protocol was approved by the Institutional Review Board of Chonnam National University Hospital and followed the tenets of the Declaration of Helsinki.

\section{Clinical data and optical coherence tomography imaging}

Factors evaluated were age at diagnosis, sex, SE refractive error, axial length, central corneal thickness, baseline IOP before treatment, family history of glaucoma, and various systemic factors including diabetes mellitus, hypertension, cold hands/feet, and migraine. Goldmann applanation tonometry was used for all IOP measurements. Central corneal thickness and axial length were measured more than three times by ultrasound pachymetry (UP-1000, Nidek Co., Ltd, Tokyo, Japan) and A-scan ultrasonography (Model US-800, Nidek Co., Ltd), respectively, at the initial visit and an average was calculated. Measurements of peripapillary RNFL (pRNFL) and macular ganglion cell-inner plexiform layer (mGCIPL) parameters were performed using a Cirrus high-definition optical coherence tomography device (Carl Zeiss Meditec).

\section{Evaluation of optic disc characteristics}

Digital retinal photographs centered on the optic disc and macula were obtained using standard settings with a nonmydriatic retinal camera. Each photograph was exported to a desktop computer as a TIFF image file. Using public domain Java-based image processing software developed by the National Institutes of Health (ImageJ, version 1.4.1; Wayne Rasband; National Institutes of Health, Rockville, MD, USA), optic disc tilt, rotation, and the optic disc area were measured by two independent examiners (SWP and MSS). Averaged data were used in the final analysis. The measurement of optic disc tilt has been previously 
described. ${ }^{15,19-21}$ Optic discs with tilt ratios exceeding 1.30 were classified as tilted.

Optic disc rotation was defined as the deviation of the long axis of the optic disc from the reference line, $90^{\circ}$ from a horizontal line connecting the fovea and the center of the optic disc. The angle between the long axis of the optic disc and the reference line was termed the degree of optic disc rotation. Superior rotation indicated clockwise rotation of the optic disc in the right eye or counterclockwise rotation of the optic disc in the left eye; inferior rotation indicated counter-clockwise rotation of the optic disc in the right eye or clockwise rotation of the optic disc in the left eye. Superior rotation was presented as a negative value, and inferior rotation was presented as a positive value. The optic disc was classified as having significant rotation when the degree of rotation exceed $15^{\circ}$.

The optic disc area was determined as the total number of pixels using ImageJ software in a circumferential pattern. Combined with a fundus camera magnification factor of $\times 1.4$, the total magnification by the camera and ImageJ system was calculated. We converted the optic disc area from pixels to $\mathrm{mm}^{2}$. The area was corrected using Littmann's formula for each axial length. ${ }^{22}$ The anterior corneal curvature radius was set at $7.8 \mathrm{~mm}$, which is the reported mean for white and Chinese persons. ${ }^{23}$ The presence of $\beta$-zone PPA and optic disc hemorrhage on the initial digital retinal photograph was also noted. $\beta$-zone PPA was defined as marked atrophy of the retinal pigment epithelium and a horizontal width of the choriocapillaris apparently larger than the diameter of the major retinal vein at the optic disc edge.

\section{Analysis of visual field}

A reliable VF was required to have a fixation loss of $<20 \%$ and a false-negative and false-positive rate of $\leq 15 \%$. A glaucomatous VF required having a cluster of $\geq 3$ contiguous test locations at $P<0.05$ on the pattern deviation plot, with $\geq 1$ test location at $P<0.01$. The results of the second test were used in the analysis to remove the learning effect. The second VF examination was performed within 1 month from the first visit. Parafoveal scotoma was defined as a VF defect in one hemifield within $10^{\circ}$ of fixation with at least one point at $P<0.01$ lying at the two innermost paracentral points, with or without defects outside the central $10^{\circ}$ in the superior or inferior arcuate area. To be classified in the parafoveal scotoma group, eyes were required to have a consistent parafoveal scotoma on two consecutive reliable 30-2 VF tests. The VF indices including MD, pattern standard deviation (PSD), and visual field index (VFI) were evaluated. In addition, average total deviation (TD) sensitivity (dB) of test points in a TD map was calculated.
Central average TD and parafoveal average TD values were defined as mean thresholds of the central innermost 4 points and parafoveal innermost 12 points, respectively. The nasal peripheral average TD value was defined as the mean thresholds of the nasal peripheral 18 points on a TD map of 30-2 VF (Supplementary Figure 1).

\section{Statistical analyses}

SPSS version 18.0 (SPSS, Chicago, IL, USA) was used for all statistical analyses. Agreement on the optic disc tilt ratio, degree of rotation, and optic disc area between two observers was assessed using the Bland-Altman method, which plots means against differences. ${ }^{24}$ The limits of agreement were defined as the mean differences of two measurements $\pm 1.96 \mathrm{SD}$ of the difference. The normality of distribution was verified using the Shapiro-Wilk normality test. Baseline characteristics were reported in counts and proportions or the mean $\pm \mathrm{SD}$ as appropriate. Groups were compared using the $X^{2}$-test, Fisher's Exact test, the independent $t$-test, or the Mann-Whitney $U$-test as appropriate. Univariate and multivariate logistic regression analyses were performed to determine the association of various factors with development of parafoveal scotoma in early myopic NTG. Variables with $P<0.10$ in the univariate model were entered in a multivariate model. In order to control for the type I error, Holm-Bonferroni-adjusted $P$-values were used. Correlations between the parameters were assessed by linear and quadratic regression analyses. A $P$-value $<0.05$ was considered to be statistically significant.

\section{Results}

The study included 99 eyes of 99 early myopic NTG patients. The mean age at the initial visit was $38.88 \pm 9.91$ years and mean SE refractive error was $-6.07 \pm 2.83 \mathrm{D}$. All subjects had single hemifield VF defects, and of 99 eyes, $42(42.42 \%)$ had parafoveal scotoma. The interobserver agreement using Bland-Altman plots in the measurements of the optic disc area, tilt ratio, and degree of optic disc rotation for all subjects showed no systematic differences in measurements.

Table 1 summarizes the clinical and demographic characteristics of included subjects. There were no statistically significant differences in age, gender, systemic factors, family history, degree of myopia, central corneal thickness, and baseline IOP between the eyes with and without parafoveal scotoma. With regard to optic disc characteristics, eyes with parafoveal scotoma had more tilted disc and presented more frequently with inferior optic disc rotation and $\beta$-zone PPA, compared with eyes without parafoveal scotoma. In addition, eyes with parafoveal scotoma were noted to have a lower 
Table 1 Comparison of the clinical characteristics between eyes with and without parafoveal scotoma in early myopic normal tension glaucoma

\begin{tabular}{|c|c|c|c|c|}
\hline Variables & All $(\mathrm{n}=99)$ & $\begin{array}{l}\text { With parafoveal } \\
\text { scotoma }(\mathrm{n}=42)\end{array}$ & $\begin{array}{l}\text { Without parafoveal } \\
\text { scotoma }(\mathrm{n}=57)\end{array}$ & P-value ${ }^{\mathrm{a}}$ \\
\hline Age at diagnosis, $\mathrm{y}$ & $38.88 \pm 9.91$ & $38.79 \pm 9.99$ & $38.95 \pm 9.93$ & 0.937 \\
\hline Male, $n(\%)$ & $57(57.58)$ & $28(66.67)$ & $29(50.88)$ & 0.116 \\
\hline \multicolumn{5}{|l|}{ Systemic factors } \\
\hline Diabetes mellitus, $n(\%)$ & $6(6.06)$ & $3(7.14)$ & $3(5.26)$ & 0.696 \\
\hline Hypertension, $n(\%)$ & $3(3.03)$ & $0(0)$ & $3(5.26)$ & 0.260 \\
\hline Cold hands/feet, $n(\%)$ & $8(8.08)$ & $3(7.14)$ & $5(8.77)$ & 0.769 \\
\hline Migraine, $n(\%)$ & $3(3.03)$ & $1(2.38)$ & $2(3.51)$ & 0.746 \\
\hline Family history, $n(\%)$ & $3(3.03)$ & $3(7.14)$ & $0(0)$ & 0.073 \\
\hline SE refractive error, D & $-6.07 \pm 2.83$ & $-6.16 \pm 2.83$ & $-6.00 \pm 2.85$ & 0.777 \\
\hline High myopia, $n(\%)$ & $45(45.45)$ & $21(50)$ & $24(42.11)$ & 0.541 \\
\hline Axial length, $\mathrm{mm}$ & $26.29 \pm 1.20$ & $26.41 \pm 1.33$ & $26.21 \pm 1.11$ & 0.554 \\
\hline Central corneal thickness, $\mu \mathrm{m}$ & $542.21 \pm 36.19$ & $537.53 \pm 38.07$ & $545.57 \pm 34.75$ & 0.299 \\
\hline Baseline IOP, mm Hg & $17.49 \pm 2.27$ & $17.48 \pm 2.05$ & $17.51 \pm 2.44$ & 0.944 \\
\hline \multicolumn{5}{|l|}{ Optic disc characteristics } \\
\hline Disc area, $\mathrm{mm}^{2}$ & $1.91 \pm 0.47$ & $1.88 \pm 0.47$ & $1.93 \pm 0.48$ & 0.627 \\
\hline Vertical CDR & $0.72 \pm 0.14$ & $0.75 \pm 0.10$ & $0.70 \pm 0.15$ & 0.087 \\
\hline Tilt ratio & $1.38 \pm 0.21$ & $1.45 \pm 0.23$ & $1.32 \pm 0.17$ & 0.002 \\
\hline Tilted disc, $n(\%)$ & $67(67.68)$ & $31(73.81)$ & $36(63.16)$ & 0.263 \\
\hline Rotation degree, ${ }^{\circ}$ & $1.75 \pm 19.59$ & $4.02 \pm 18.27$ & $0.07 \pm 20.50$ & 0.324 \\
\hline Significant optic disc rotation, $n(\%)$ & $49(49.49)$ & $13(30.95)$ & $36(63.16)$ & 0.002 \\
\hline Inferior rotation, $n(\%)$ & $56(56.57)$ & $31(73.81)$ & $25(43.86)$ & 0.003 \\
\hline$\beta$-zone PPA, $n(\%)$ & $73(73.74)$ & $36(85.71)$ & $37(64.91)$ & 0.020 \\
\hline Optic disc hemorrhage, $n(\%)$ & $10(10.10)$ & $6(14.29)$ & $4(7.02)$ & 0.316 \\
\hline \multicolumn{5}{|l|}{ OCT measurement, $\mu \mathrm{m}$} \\
\hline Average pRNFL thickness & $73.82 \pm 10.48$ & $71.81 \pm 10.69$ & $75.30 \pm 10.15$ & 0.102 \\
\hline Superior pRNFL thickness & $88.37 \pm 16.78$ & $88.50 \pm 16.78$ & $88.28 \pm 16.92$ & 0.949 \\
\hline Inferior pRNFL thickness & $80.21 \pm 19.03$ & $77.02 \pm 15.94$ & $82.56 \pm 20.85$ & 0.153 \\
\hline Average mGCIPL thickness & $69.25 \pm 8.26$ & $66.55 \pm 8.98$ & $71.25 \pm 7.12$ & 0.005 \\
\hline Minimum mGCIPL thickness & $59.98 \pm 10.08$ & $55.48 \pm 8.73$ & $63.30 \pm 9.78$ & $<0.001$ \\
\hline
\end{tabular}

Abbreviations: $\mathrm{CDR}=$ cup-to-disc ratio; $\mathrm{D}$, diopters; IOP, intraocular pressure; mGCIPL, macular ganglion cell-inner plexiform layer; OCT, optical coherence tomography; PPA, parapapillary atrophy; $\mathrm{PRNFL}$, peripapillary retinal nerve fiber layer; SE, spherical equivalent. ${ }^{\mathrm{a}} \mathrm{P}$-value for comparisons between the eyes with and without parafoveal scotoma groups using independent $t$-test, $X^{2}$-test, or Fisher's exact test, as appropriate.

Table 2 Comparison of perimetric parameters between eyes with and without parafoveal scotoma in early myopic normal tension glaucoma

\begin{tabular}{lccrr}
\hline Perimetric parameters & All $(\mathrm{n}=99)$ & With parafoveal scotoma $(\mathrm{n}=42)$ & Without parafoveal scotoma $(\mathrm{n}=57)_{\text {P-value }}{ }^{2}$ \\
\hline MD, dB & $-3.29 \pm 1.70$ & $-3.75 \pm 1.72$ & $-2.94 \pm 1.62$ & 0.018 \\
PSD, dB & $6.08 \pm 2.97$ & $6.14 \pm 2.98$ & $6.03 \pm 2.99$ & 0.859 \\
VFI, \% & $91.24 \pm 5.45$ & $88.29 \pm 5.68$ & $93.42 \pm 4.12$ & $<0.001$ \\
Central average TD, dB & $-3.10 \pm 4.08$ & $-6.40 \pm 4.30$ & $-0.67 \pm 1.20$ & $<0.001$ \\
Parafoveal average TD, dB & $-4.03 \pm 3.52$ & $-6.56 \pm 3.20$ & $-2.17 \pm 2.44$ & $<0.001$ \\
Nasal peripheral average TD, dB & $-5.87 \pm 4.31$ & $-5.25 \pm 4.01$ & $-6.32 \pm 4.50$ & $<0.001$ \\
Superior defect, $n(\%)$ & $68(68.69)$ & $36(85.71)$ & $32(56.14)$ & 0.007 \\
\hline
\end{tabular}

Abbreviations: dB, decibel; MD, mean deviation; PSD, pattern standard deviation; TD, total deviation; VFI, visual field index. ${ }^{\text {a }}$-value for comparison between the eyes with and without parafoveal scotoma groups using independent $t$-test, $X^{2}$-test, or Fisher's exact test, as appropriate.

prevalence of significant optic disc rotation and thinner average, and minimum mGCIPL thickness. Table 2 shows the results of VF examinations in the study subjects. All perimetric parameters except the PSD value were significantly different between the two groups. On multivariate logistic regression analysis, tilt ratio, significant optic disc rotation, minimum mGCIPL thickness, baseline MD, and VF defects in the superior 
Table 3 Logistic regression analysis with the dependent variable being the development of parafoveal scotoma in early myopic normal tension glaucoma

\begin{tabular}{|c|c|c|c|c|}
\hline \multirow[t]{2}{*}{ Variables } & \multicolumn{2}{|c|}{ Univariate } & \multicolumn{2}{|c|}{ Multivariate ${ }^{\mathrm{a}}$} \\
\hline & Odds ratio $(95 \% \mathrm{CI})$ & P-value & Odds ratio $(95 \% \mathrm{CI})$ & P-value \\
\hline Vertical CDR & $16.976(0.624-461.532)$ & 0.093 & & \\
\hline Tilt ratio & $27.268(2.913-255.279)$ & 0.004 & $16.060(1.022-252.287)$ & 0.047 \\
\hline Significant optic disc rotation & $0.261(0.112-0.610)$ & 0.002 & $0.259(0.091-0.734)$ & 0.011 \\
\hline Inferior rotation & $3.607(1.520-8.560)$ & 0.004 & & \\
\hline$\beta$-zone PPA & $3.243(1.168-9.005)$ & 0.024 & & \\
\hline Average mGCIPL thickness, $\mu \mathrm{m}$ & $0.928(0.879-0.980)$ & 0.007 & & \\
\hline Minimum mGCIPL thickness, $\mu \mathrm{m}$ & $0.912(0.867-0.959)$ & $<0.001$ & $0.920(0.863-0.981)$ & 0.010 \\
\hline Baseline $\mathrm{MD}, \mathrm{dB}$ & $0.745(0.581-0.955)$ & 0.020 & $0.699(0.504-0.969)$ & 0.032 \\
\hline VF defect on superior hemifield & $4.687(1.707-12.874)$ & 0.003 & $8.518(2.309-31.421)$ & 0.001 \\
\hline
\end{tabular}

Abbreviations: CI, confidence interval; CDR, cup-to-disc ratio; MD, mean deviation; mGCIPL, macular ganglion cell-inner plexiform layer; PPA, parapapillary atrophy; VF, visual field. Variables with $P>0.10$ in the univariate model are not shown in this table. Variables with $P<0.10$ in the univariate model were entered in a multivariate model. ${ }^{a}$ Variables were entered in the model if $P<0.05$ and removed if $P>0.10$ in the saturated multivariate model.

hemifield were all independently associated with development of parafoveal scotoma in early myopic NTG (Table 3).

As our main interest was to investigate the association between myopic optic disc characteristics and the development of parafoveal scotoma, we classified myopic NTG patients based on the presence of optic disc tilt and rotation. Sixty-seven $(67.68 \%)$ eyes were classified into a tilted disc group and 32 (32.32\%) eyes into a non-tilted disc group. In the tilted disc group, eyes without significant optic disc rotation exhibited more severe parafoveal VF defects as determined by worse central and parafoveal average TD and thinner average, and minimum mGCIPL. In the non-tilted disc group, no statistically significant difference was observed in any perimetric or OCT parameter between eyes with and without significant optic disc rotation (Supplementary Table 4).

The scatter plots in Figure 1 show the association between the tilt ratio and parameters of parafoveal scotoma mean depth defined as central and parafoveal average TD values. Linear and quadratic regression analyses demonstrated that a higher degree of optic disc tilt was related to a worse central average TD and parafoveal average TD (Figures 1a and d). The relationships were stronger in eyes without significant optic disc rotation (Figures $1 \mathrm{~b}$ and e). But, there was no statistically significant relationship between the tilt ratio and parafoveal scotoma mean depth among eyes with significant optic disc rotation (Figures $1 \mathrm{c}$ and $\mathrm{f}$ ).

\section{Discussion}

As morphological changes in the optic disc have been significantly associated with the development of NTG in myopic eyes, ${ }^{12-15}$ in the present study we hypothesized that anatomic changes in the optic disc might be associated with the pattern of VF defect in myopic NTG. In early myopic NTG, we found that a thinner minimum mGCIPL, worse baseline MD, and a VF defect in the superior hemifield were associated with parafoveal scotoma. In addition, the optic disc tilt ratio and significant optic disc rotation were significantly associated with the development of parafoveal scotoma. We found that the higher the degree of optic disc tilt, the worse the parafoveal VF defect. Significant optic disc rotation had a protective role against the development of parafoveal scotoma in eyes with optic disc tilt. However, in contrast to previous reports, the degree of myopia, baseline IOP, and systemic factors did not show any significant association with the parafoveal scotoma. $3,18,25,26$ To the best of our knowledge, this is the first study evaluating risk factors for parafoveal scotoma in myopic NTG patients.

Among the included eyes, $45.45 \%$ showed high myopia and nearly half (42.42\%) showed parafoveal scotoma in the early disease course. Given that parafoveal scotoma is a relatively common finding in eyes with NTG and high myopia, the high prevalence of parafoveal scotoma is not surprising. ${ }^{18,25-29}$ Earlier studies showed that glaucomatous VF defects of NTG are more centrally located than those of high tension glaucoma, ${ }^{28,29}$ and the association between high myopia and the risk of parafoveal scotoma in glaucoma has also been previously reported. ${ }^{18,25-27}$ However, in contrast to previous studies demonstrating high myopia to be a risk factor for parafoveal scotoma or papillomacular bundle defect, ${ }^{18,25,27}$ we did not find significant associations between SE refractive error, axial length, or high myopia and parafoveal scotoma on regression analysis. Although our results are not directly comparable with earlier studies because of different study designs and populations, the lack of association suggests that there 

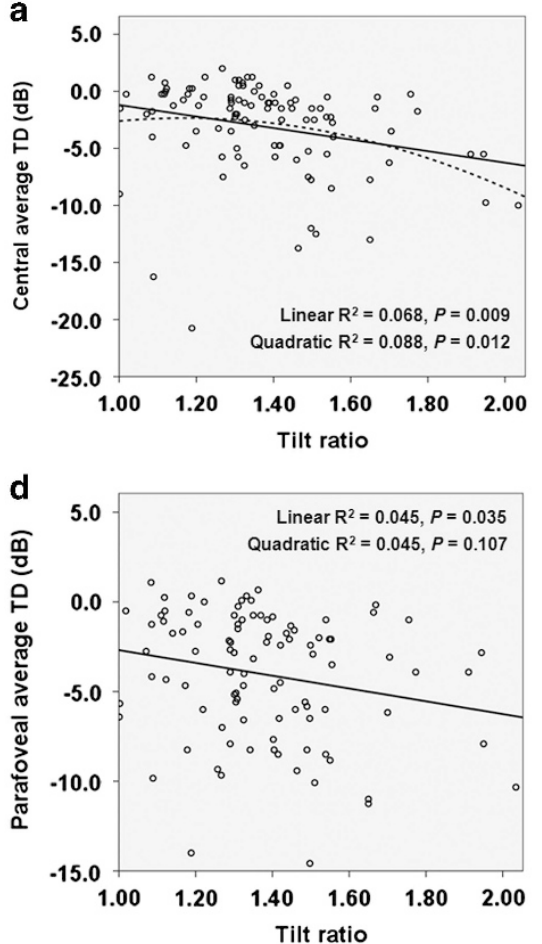

b
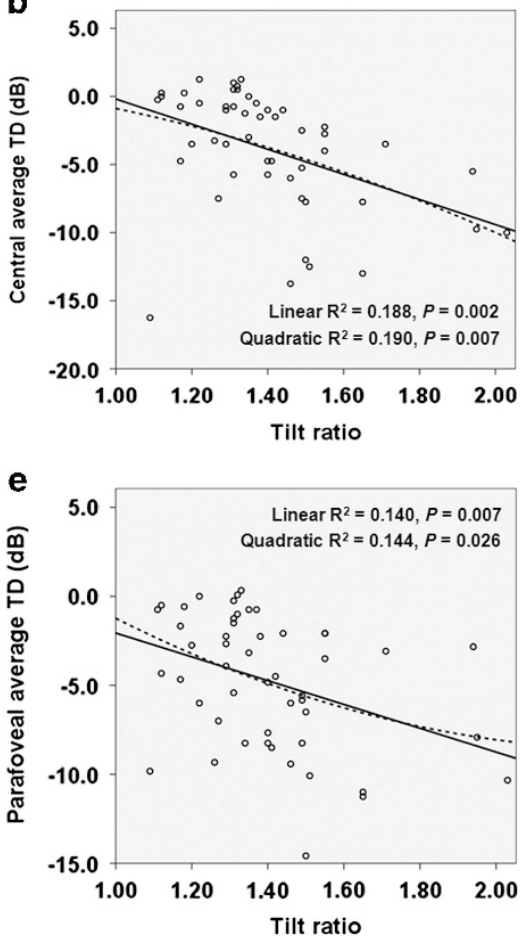

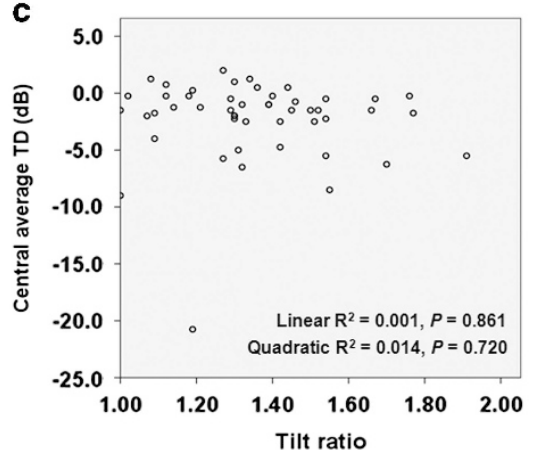

$f$

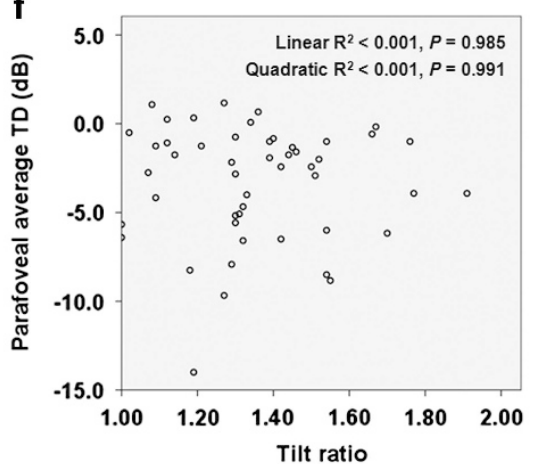

Figure 1 Scatter plots showing the relationship between the severity of parafoveal scotoma and tilt ratio. (a, d) The relationship between central average TD (a) and parafoveal average TD (d) values and tilt ratio in 99 early myopic NTG eyes. (b, e) The relationship between central average TD (b) and parafoveal average TD (e) values, and tilt ratio in 50 early myopic NTG eyes without significant optic disc rotation. (c, f) The relationship between central average TD (c) and parafoveal average TD (f) values, and tilt ratio in 49 early myopic NTG eyes with significant optic disc rotation. $P$-values for all linear and quadratic regression are presented.

may be other factors involved in the interaction between myopia and development of a parafoveal scotoma.

In the comparison of perimetric parameters, MD and VFI were significantly worse in eyes with parafoveal scotoma. Two possible explanations might account for our results. First, both MD and VFI are more centrally weighted values. Second, because all eyes with parafoveal VF defect were assigned to the parafoveal scotoma group, regardless of whether or not they presented with an additional peripheral VF defect, the proportion of patients with a large VF defect in eyes with parafoveal scotoma might have been higher than that in eyes without parafoveal scotoma. It should be noted that the prevalence of parafoveal scotoma increases with disease progression. The result of our regression analysis demonstrating that baseline MD is a risk factor for parafoveal scotoma might be similarly explained. In terms of the location of VF defects, parafoveal scotoma was found more frequently in the superior hemifield. The unequal prevalence of parafoveal scotoma between hemifields has been extensively investigated and our results are consistent with those of previous reports. $3,17,18,30-34$

It was reported that macular structural parameters using spectral-domain OCT may be potentially useful predictors of the presence of parafoveal scotoma. ${ }^{35-37}$ In accordance with the previous findings, our current results showed that the average and minimum mGCIPL thickness in eyes with parafoveal scotoma was significantly thinner than in eyes without parafoveal scotoma. However, the pRNFL thickness parameters were not significantly different between the two groups. These findings might be attributable to the anatomic differences between pRNFL and mGCIPL. Because all retinal ganglion cell axons gather to the optic disc, pRNFL thickness measurements centered at the optic disc cannot indicate retinal ganglion cell loss in a specific region. In contrast, mGCIPL thickness measurements reflect retinal ganglion cells within the central retina. Thus, thinning of the mGCIPL may be more evident in eyes with parafoveal scotoma.

In the multivariate logistic regression analysis, we found that optic disc tilt significantly influenced the risk of parafoveal scotoma in eyes with early myopic NTG. It has been shown that tilted optic disc is the consequence of progressive stretching of the sclera during myopic shift. ${ }^{38}$ As the eyeball grows axially, the temporal sclera moves back and becomes flattened. We postulate that these changes make the temporal side of the lamina cribrosa, which is the principal site of axonal damage in glaucoma, 
more susceptible to mechanical strain and these processes result in damage to the papillomacular bundle. Therefore, the papillomacular bundle might be vulnerable to glaucomatous damage in myopic eyes even before superior and inferior arcuate bundle damage. The OCT study by Kimura et $a l^{39}$ that evaluated optic disc morphology in highly myopic eyes supports our speculation. They reported that the presence of defects in the lamina cribrosa was significantly associated with the degree of vertical disc tilt. They also found that disinsertions of the lamina cribrosa were located in the temporal quadrant in eyes with high myopia.

Of note, we found significant interaction between optic disc tilt and optic disc rotation for developing parafoveal scotoma. When we performed subgroup analysis based on the presence of optic disc tilt and rotation, in eyes with non-tilted disc, optic disc rotation did not show any significant effect on the risk for a parafoveal scotoma. However, significant optic disc rotation reduced the risk of a parafoveal scotoma in eyes with tilted disc. As shown in the representative two cases in Figure 2, the patterns of VF defect differed noticeably according to the presence of significant optic disc rotation, although they had a similar degree of myopia, MD, and tilt ratio.

The clinical implications of optic disc rotation in glaucoma has been well established. ${ }^{14,15,21}$ However, the mechanism underlying the development of optic disc rotation has not been clearly understood. Traditionally, it was believed that optic disc rotation is caused by a real rotation of the disc during the axial elongation. However, Lee et $a l^{40}$ have recently suggested that optic disc rotation is another form of disc tilt centered on the oblique axis. If the former suggestion is correct, the original temporal site of the lamina cribrosa containing the papillomacular bundle might be still subject to additional mechanical strain by myopic tilting, even if the optic disc is rotated during axial elongation. Considering that the receptive field in the retina corresponding to the papillomacular bundle is constant, a tilted optic disc would show a similar pattern of VF damage regardless of optic disc rotation. Accordingly, eyes with tilted optic disc accompanying significant disc rotation would also show a high prevalence of central VF defects similar to eyes with tilted optic disc accompanying minimal disc rotation.

However, this was not the case in our study. Among the 21 eyes that had tilted optic disc with significant inferior rotation, 17 (80.95\%) showed inferior RNFL damage and only 7 (33.33\%) showed parafoveal scotoma. We suggest that our result provides additional support for the notion that optic disc rotation is just an anatomic finding resulting from oblique disc tilt rather than a result of real rotation. For example, in the case of inferior rotation, the optic disc may tilt inferiorly on an oblique axis and the site of maximal mechanical strain may not be the
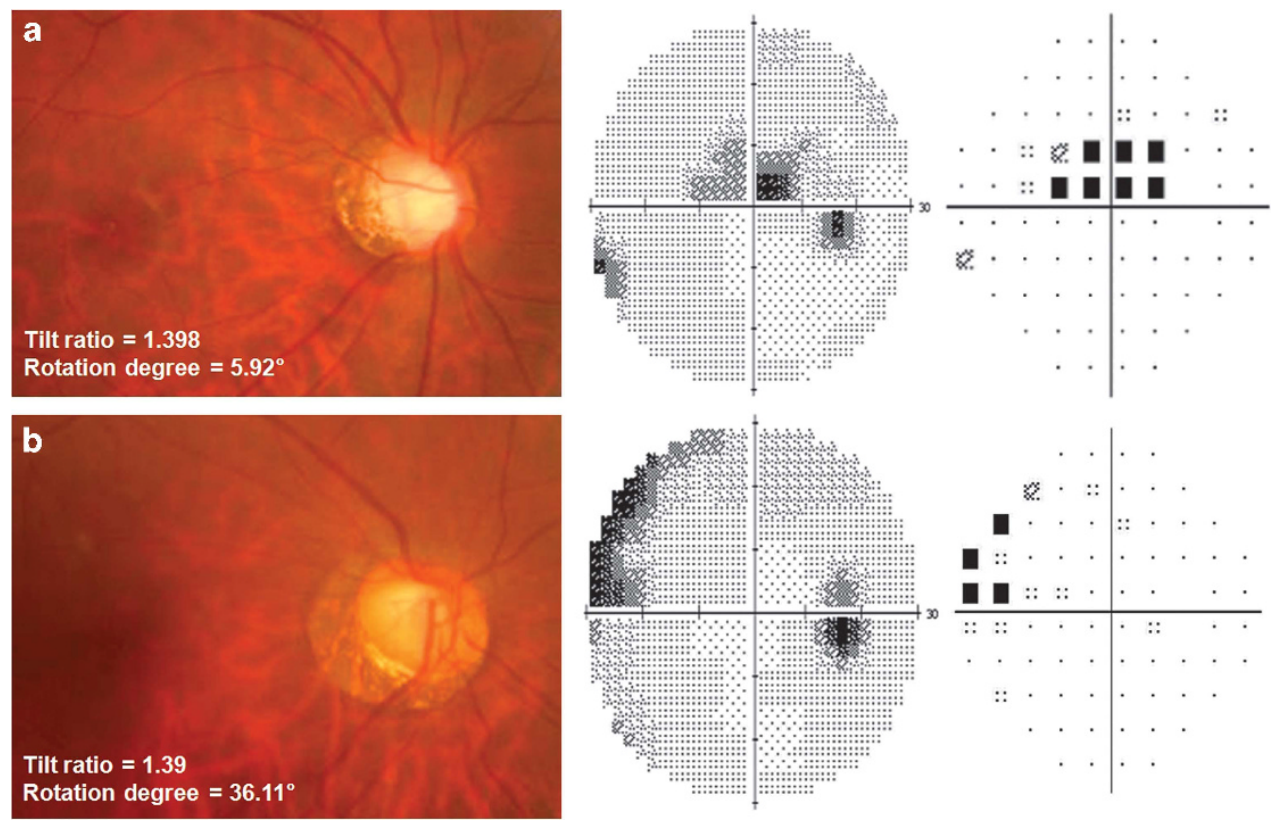

Figure 2 Two representative cases of myopic normal tension glaucoma with similar tilt ratio and different degrees of optic disc rotation and their pattern of visual field defect on gray scale and pattern deviation plot. (a) Images from a 39-year-old man with parafoveal scotoma (SE refractive error $=-9.25 \mathrm{D}$, axial length $=27.55 \mathrm{~mm}, \mathrm{MD}=-0.55 \mathrm{~dB}, \mathrm{PSD}=5.09 \mathrm{~dB}$ ). (b) Images from a 47 -year-old woman without parafoveal scotoma (SE refractive error $=-8.00 \mathrm{D}$, axial length $=27.01 \mathrm{~mm}, \mathrm{MD}=-3.34 \mathrm{~dB}, \mathrm{PSD}=4.65 \mathrm{~dB}$ ). The patterns of visual field defect differed noticeably according to the presence of significant optic disc rotation, although they had a similar degree of myopia, MD, and tilt ratio. 
temporal part of the lamina cribrosa but the more inferior part of the lamina cribrosa.

There are also several limitations to this study. First, it was conducted in Korea and contained a relatively small number of subjects, so our findings may not be generalizable to other ethnic groups. Second, the crosssectional nature of our study limited our ability to describe the temporal relationships of optic disc tilt, rotation, and the development of central VF progression. Future prospective, longitudinal studies are needed to understand better the role of optic disc tilt and rotation in the development of parafoveal scotoma. Third, we did not measure the actual optic disc tilt, and instead used the optic disc ovality index. However, disc ovality has been used as a surrogate index of disc tilt. ${ }^{41,42}$ Finally, our study sample included patients from a tertiary referral center, so there might be an element of selection bias, that is, the prevalence of parafoveal scotoma might be exaggerated.

In conclusion, in this study we identified factors associated with parafoveal scotoma in patients with myopic NTG and found that tilted optic discs without significant rotation are at risk for early central VF involvement. Given the importance of central VF in patients with glaucoma, optic discs at risk should receive more attention and aggressive management. In this regard, our current study might help clinicians to establish guidelines for managing patients with myopic NTG.

\section{Summary}

What was known before

- In myopic eyes, morphological changes in the optic disc induced by axial elongation, such as $\beta$-zone parapapillary atrophy, tilt, and rotation, have an important role in the development of glaucoma.

- Parafoveal scotoma is a relatively common finding in eyes with normal tension glaucoma (NTG) and myopia. However, factors associated with the development of parafoveal scotoma in eyes with myopic NTG have not been reported.

What this study adds

- In patients with myopic NTG, tilted optic discs without significant rotation are at risk for early central visual field involvement.

\section{Conflict of interest}

The authors declare no conflict of interest.

\section{Author contributions}

All authors had full access to all the data in the study and take responsibility for the integrity of the data and accuracy of the data analyses.

\section{Acknowledgements}

This research was supported by the Basic Science Research program through the National Research Foundation of Korea funded by the Ministry of Education (NRF-2015R1D1A1A01059630), Seoul, Korea. The funding organizations had no role in the design or conduct of this research.

\section{References}

1 Kolker AE. Visual prognosis in advanced glaucoma: a comparison of medical and surgical therapy for retention of vision in 101 eyes with advanced glaucoma. Trans Am Ophthalmol Soc 1977; 75: 539-555.

2 Coeckelbergh TR, Brouwer WH, Cornelissen FW, Van Wolffelaar P, Kooijman AC. The effect of visual field defects on driving performance: a driving simulator study. Arch Ophthalmol 2002; 120(11): 1509-1516.

3 Park SC, De Moraes CG, Teng CC, Tello C, Liebmann JM, Ritch R. Initial parafoveal versus peripheral scotomas in glaucoma: risk factors and visual field characteristics. Ophthalmology 2011; 118(9): 1782-1789.

4 Anctil JL, Anderson DR. Early foveal involvement and generalized depression of the visual field in glaucoma. Arch Ophthalmol 1984; 102(3): 363-370.

5 Stamper RL. The effect of glaucoma on central visual function. Trans Am Ophthalmol Soc 1984; 82: 792-826.

6 Park HY, Jung KI, Na KS, Park SH, Park CK. Visual field characteristics in normal-tension glaucoma patients with autonomic dysfunction and abnormal peripheral microcirculation. Am J Ophthalmol 2012; 154(3): 466-475.

7 Lee M, Cho EH, Lew HM, Ahn J. Relationship between ocular pulse amplitude and glaucomatous central visual field defect in normal-tension glaucoma. J Glaucoma 2012; 21(9): 596-600.

8 Levene RZ. Low tension glaucoma: a critical review and new material. Surv Ophthalmol 1980; 24(6): 621-664.

9 Thonginnetra O, Greenstein VC, Chu D, Liebmann JM, Ritch R, Hood DC. Normal versus high tension glaucoma: a comparison of functional and structural defects. J Glaucoma 2010; 19(3): 151-157.

10 Ahrlich KG, De Moraes CG, Teng CC, Prata TS, Tello C, Ritch $\mathrm{R}$ et al. Visual field progression differences between normal-tension and exfoliative high-tension glaucoma. Invest Ophthalmol Vis Sci 2010; 51(3): 1458-1463.

11 Kim DM, Seo JH, Kim SH, Hwang SS. Comparison of localized retinal nerve fiber layer defects between a low-teen intraocular pressure group and a high-teen intraocular pressure group in normal-tension glaucoma patients. J Glaucoma 2007; 16(3): 293-296.

12 Teng CC, De Moraes CG, Prata TS, Liebmann CA, Tello $C$, Ritch $\mathrm{R}$ et al. The region of largest $\beta$-zone parapapillary atrophy area predicts the location of most rapid visual field progression. Ophthalmology 2011; 118(12): 2409-2413.

13 Choi JA, Park HY, Shin HY, Park CK. Optic disc tilt direction determines the location of initial glaucomatous damage. Invest Ophthalmol Vis Sci 2014; 55(8): 4991-4998.

14 Park HY, Lee KI, Lee K, Shin HY, Park CK. Torsion of the optic nerve head is a prominent feature of normal-tension glaucoma. Invest Ophthalmol Vis Sci 2014; 56(1): 156-163. 
15 Park HY, Lee K, Park CK. Optic disc torsion direction predicts the location of glaucomatous damage in normaltension glaucoma patients with myopia. Ophthalmology 2012; 119(9): 1844-1851.

16 Lee M, Jin H, Ahn J. Relationship between disc margin to fovea distance and central visual field defect in normal tension glaucoma. Graefes Arch Clin Exp Ophthalmol 2014; 252(2): 307-314

17 Choi JA, Park HY, Park CK. Difference in the posterior pole profiles associated with the initial location of visual field defect in early-stage normal tension glaucoma. Acta Ophthalmol 2015; 93(2): e94-e99.

18 Kimura Y, Hangai M, Morooka S, Takayama K, Nakano N, Nukada $\mathrm{M}$ et al. Retinal nerve fiber layer defects in highly myopic eyes with early glaucoma. Invest Ophthalmol Vis Sci 2012; 53(10): 6472-6478

19 Lee KS, Lee JR, Kook MS. Optic disc torsion presenting as unilateral glaucomatous-appearing visual field defect in young myopic Korean eyes. Ophthalmology 2014; 121(5): 1013-1019.

20 Sung MS, Kang YS, Heo H, Park SW. Characteristics of optic disc rotation in myopic eyes. Ophthalmology 2016; 123(2): 400-407.

21 Sung MS, Kang YS, Heo H, Park SW. Optic disc rotation as a clue for predicting visual field progression in myopic normal-tension glaucoma. Ophthalmology 2016; 123(7): 1484-1493.

22 Bennett AG, Rudnicka AR, Edgar DF. Improvements on Littmann's method of determining the size of retinal features by fundus photography. Graefes Arch Clin Exp Ophthalmol 1994; 232(6): 361-367.

23 Cheung SW, Cho P, Douthwaite W. Corneal shape of Hong Kong-Chinese. Ophthalmic Physiol Opt 2000; 20(2): 119-125.

24 Bland JM, Altman DG. Statistical methods for assessing agreement between two methods of clinical measurement. Lancet 1986; 1(8476): 307-310.

25 Kim JM, Park KH, Kim SJ, Jang HJ, Noh E, Kim MJ et al. Comparison of localized retinal nerve fiber layer defects in highly myopic, myopic, and non-myopic patients with normal-tension glaucoma: a retrospective cross-sectional study. BMC Ophthalmol 2013; 13: 67.

26 Mayama C, Suzuki Y, Araie M, Ishida K, Akira T, Yamamoto $\mathrm{T}$ et al. Myopia and advanced-stage open-angle glaucoma. Ophthalmology 2002; 109(11): 2072-2077.

27 Araie M, Arai M, Koseki N, Suzuki Y. Influence of myopic refraction on visual field defects in normal tension and primary open angle glaucoma. Jpn J Ophthalmol 1995; 39(1): 60-64.

28 Caprioli J, Spaeth GL. Comparison of visual field defects in the low-tension glaucomas with those in the high-tension glaucomas. Am J Ophthalmol 1984; 97(6): 730-737.
29 Chihara E, Tanihara H. Parameters associated with papillomacular bundle defects in glaucoma. Graefes Arch Clin Exp Ophthalmol 1992; 230(6): 511-517.

30 Choi JA, Park HY, Jung KI, Hong KH, Park CK. Difference in the properties of retinal nerve fiber layer defect between superior and inferior visual field loss in glaucoma. Invest Ophthalmol Vis Sci 2013; 54(10): 6982-6990.

31 Hood DC, Raza AS, de Moraes CG, Odel JG, Greenstein VC, Liebmann JM et al. Initial arcuate defects within the central 10 degrees in glaucoma. Invest Ophthalmol Vis Sci 2011; 52(2): 940-946.

32 Hood DC, Slobodnick A, Raza AS, de Moraes CG, Teng CC, Ritch R. Early glaucoma involves both deep local, and shallow widespread, retinal nerve fiber damage of the macular region. Invest Ophthalmol Vis Sci 2014; 55(2): 632-649.

33 Jung KI, Park HY, Park CK. Characteristics of optic disc morphology in glaucoma patients with parafoveal scotoma compared to peripheral scotoma. Invest Ophthalmol Vis Sci 2012; 53(8): 4813-4820.

34 Hood DC, Raza AS, de Moraes CG, Liebmann JM, Ritch R. Glaucomatous damage of the macula. Prog Retin Eye Res 2013; 32: 1-21.

35 Kimura Y, Hangai M, Matsumoto A, Akagi T, Ikeda HO, Ohkubo $\mathrm{S}$ et al. Macular structure parameters as an automated indicator of paracentral scotoma in early glaucoma. Am J Ophthalmol 2013; 156(5): 907-917.

36 Park JW, Jung HH, Heo H, Park SW. Validity of the temporal-to-nasal macular ganglion cell-inner plexiform layer thickness ratio as a diagnostic parameter in early glaucoma. Acta Ophthalmol 2015; 93(5): e356-e365.

37 Shin HY, Park HY, Jung KI, Choi JA, Park CK. Glaucoma diagnostic ability of ganglion cell-inner plexiform layer thickness differs according to the location of visual field loss. Ophthalmology 2014; 121(1): 93-99.

38 Kim TW, Kim M, Weinreb RN, Woo SJ, Park KH, Hwang JM. Optic disc change with incipient myopia of childhood. Ophthalmology 2012; 119(1): 21-26.

39 Kimura Y, Akagi T, Hangai M, Takayama K, Hasegawa T, Suda $\mathrm{K}$ et al. Lamina cribrosa defects and optic disc morphology in primary open angle glaucoma with high myopia. PLoS One 2014; 9(12): e115313.

40 Lee KM, Lee EJ, Kim TW. Lamina cribrosa configuration in tilted optic discs with different tilt axes: a new hypothesis regarding optic disc tilt and torsion. Invest Ophthalmol Vis Sci 2015; 56(5): 2958-2967.

41 Tay E, Seah SK, Chan SP, Lim AT, Chew SJ, Foster PJ et al. Optic disk ovality as an index of tilt and its relationship to myopia and perimetry. Am J Ophthalmol 2005; 139(2): 247-252.

42 Jonas JB, Kling F, Gründler AE. Optic disc shape, corneal astigmatism, and amblyopia. Ophthalmology 1997; 104(11): 1934-1937.

Supplementary Information accompanies this paper on Eye website (http://www.nature.com/eye) 